\title{
La place du médecin généraliste en psycho-oncologie
}

\author{
The role of the general practitioner in psycho-oncology
}

\author{
N. Pélicier \\ (C) Springer-Verlag France 2012
}

Le Plan cancer 2 a restitué la place occupée par le médecin généraliste dans la réalité des soins apportés aux patients atteints de cancer. Cette place incontournable suppose d'améliorer l'organisation du parcours de soin : c'est un des objets du parcours personnalisé de soins (PPS).

Comment faire connaître à nos collègues le travail des psys en cancérologie, comment peuvent-ils nous solliciter, sur quelles difficultés ?

C'est ce que nous souhaitions découvrir dans ce numéro.

Les enquêtes récentes de la Ligue nationale contre le cancer auprès des médecins généralistes [4] ont montré d'une part leur souhait d'être formés en oncologie pour mieux cerner les objectifs des soins et d'autre part leur demande d'être aidés devant l'importance de la détresse psychologique des patients qui impacte leur propre disponibilité [3].

Ce numéro voulait s'inscrire dans une présentation de l'état des lieux des pratiques du médecin généraliste à propos du soutien aux patients cancéreux, de leurs proches.

L'idée était et reste, à travers certains articles obtenus et d'autres attendus pour de prochaines parutions, d'approcher des pratiques propres aux médecins généralistes, dans la dimension de soutien qui est la leur et de repérer leurs besoins en termes d'aide et d'expertise en psychooncologie.

Le manque de contact habituel des psys avec les médecins généralistes ou leurs associations nous force à reconnaître la difficulté qui fut la nôtre pour obtenir « leur » contribution.

Le réseau ville-hôpital [1,2] intéresse le champ de la psycho-oncologie d'une part et la SFPO d'autre part depuis une dizaine d'années. Cette courte temporalité explique notre embarras. Par ailleurs, la leçon est tirée, de la nécessité de nouer et d'entretenir des relations pérennes et compréhensives

\section{N. Pélicier $(\bowtie)$}

Unité de psycho-oncologie de l'HEGP,

20, rue Leblanc, F-75908 Paris cedex 15, France

e-mail : nicole.pelicier@egp.aphp.fr avec ces partenaires indispensables que sont les médecins généralistes.

L'intérêt est bien sûr celui de la connaissance spécifique apportée par des cliniciens constamment au contact des patients et parfois de leurs familles dans un «Au dehors de l'institution de soins cancérologiques », redécouvert, entre autres, par les soins de support.

Le domicile, terrain privilégié des équipes de réseaux, reste même si cela est de plus en plus difficile en termes d'exercice le champ clinique spécifique du médecin généraliste.

Depuis peu, des liens transversaux se créent avec l'hôpital, la clinique, le CLCC, mais on attend avec impatience les résultats et les bénéfices du PPS permettant tant au patient qu'à son médecin traitant de se sortir de leur isolement dans les situations complexes qui émaillent le parcours du cancer.

Les psys, qu'ils soient « localisés » dans le dispositif d'annonce, dans l'adhésion à un réseau de soins cancérologiques ou palliatifs, dans un service ou une unité de psychooncologie, ont à repérer la démarche propre au médecin généraliste.

Peu d'études concernent précisément la place du médecin généraliste en psycho-oncologie, encore moins leurs attentes vis-à-vis des professionnels du soin psychique. Nous verrons comment les professionnels de santé expriment leurs besoins d'intervention des psys. Nous aurons aussi le témoignage particulier, presque paradoxal, de l'accompagnement d'une fin de vie, à l'image d'une vie entière, réfléchie sans difficultés particulières.

Entre le souhait que tout aille bien avec un minimum de moyens et d'interventions et l'espoir, idéalisé, que les psys résolvent les problèmes des patients et des soignants, il y a toute la nuance que doit apporter la démarche psychooncologique.

Un champ s'ouvre vers nos collèges libéraux depuis quelques années pour rendre visible nos interventions et tisser des collaborations. Il nous faut aussi nous inscrire dans une proposition de formation à la psycho-oncologie, ce à quoi travaille la SFPO, mais beaucoup reste à faire ! 


\section{Références}

1. Ellien F, Pelicier N (2008) État des lieux des organisations de la prise en charge psychologique dans les réseaux et recommandations de la SFPO. Oncologie 10(12):740-8

2. Ellien F, Pelicier N (2009) État des lieux des organisations de la prise en charge psychologique dans les réseaux et recommandations de la SFPO. Psycho-Oncol. 3(1):47-54
3. Pelicier N (2010) Enjeux psychologiques de l'après-maladie : représentations du médecin généraliste. Concours Médical

4. www.ligue-cancer.net : première rencontres Ligue nationale contre le cancer/médecins généralistes 07/10/2010 